\title{
Antimicrobial Properties of Pleurotus Eryngii and Lentinus Edodes Hydro-Alcoholic Extracts
}

\author{
Gabriela POPA $^{1,2}$, Catalina VOAIDES ${ }^{1,2}$, Petruta CORNEA ${ }^{1}$ and Valentin ZAGREAN ${ }^{3}$ \\ ${ }^{1}$ UASVM - Bucharest, Faculty of Biotechnology, Romania \\ ${ }^{2}$ Center of Applied Biochemistry and Biotechnology, BIOTEHNOL, Bucharest, Romania \\ ${ }^{3}$ ICDL-Vidra, Romania \\ * corresponding author: popagabiro@yahoo.com
}

Bulletin UASVM Animal Science and Biotechnologies 73(2)/ 2016

Print ISSN 1843-5262; Electronic ISSN 1843-536X

DOI:10.15835/buasvmcn-asb: 12284

\begin{abstract}
Besides superior nutritional values mushrooms posed significant medicinal properties. Hydro-alcoholic extracts of several isolates of Pleurotus eryngii and Lentinus edodes mushroom species were investigated for their antimicrobial activities against pathogenic microorganisms with medicinal importance. Antimicrobial activities of the extracts were evaluated by the agar disk diffusion method. Results revealed that the $70 \%$ ethylic alcohol extracts have significant inhibitory activities against Bacillus subtilis var. spizizinii, Escherichia coli and Staphylococcus aureus. The results showed that the $70 \%$ ethanol extracts of Pleurotus eryngii and Lentinus edodes mushroom isolates may have biopharmaceutical potentiality.
\end{abstract}

Keywords: antimicrobial activity, hydro-alcoholic extracts, Pleurotus eryngii, Lentinus edodes

\section{INTRODUCTION}

Several medicinal mushrooms like Lentinula spp and Pleurotus spp. are rich sources of natural antibiotics. Lentinula edodes (shiitake) extract can improve the beneficial intestinal flora, reducing harmful effects of certain bacterial diseases and colon cancer formation. Many P. eryngii extracts perform a significant effect on anticancer, antiviral, hypolipidemic and immuno-stimulating activities through immune regulatory factors (Zhiming et al., 2016). The research aims were to find natural products with antimicrobial activities which would be able to replace some synthetic antibiotics in the treatment of various infectious diseases. The objective of this work was to evaluate the antimicrobial activities of some isolates of Pleurotus eryngii and Lentinus edodes mushroom species against pathogenic microorganisms with medicinal importance.

\section{MATERIALS AND METHODS}

Four isolates of Pleurotus eryngii named Peg, Pe1, Pe 2 and P. eryngii 2600, and four isolates of Lentinus edodes named Lem 51, Lem 52, Leb and
Les were tested for their antimicrobial properties. Extracts preparation was performed by using $2 \mathrm{ml} \mathrm{70 \%} \mathrm{ethyl} \mathrm{alcohol} \mathrm{added} \mathrm{to} 1 \mathrm{~g}$ of dried mushroom sample. The hydro-alcoholic solutions were kept 18 days at the room temperature $\left(\sim 25^{\circ} \mathrm{C}\right)$.Antimicrobial activities of the extracts against Candida albicans ATCC10321, Candida parapsilopsis CBS604, Escherichia coli ATCC8739, Staphylococcus aureus ATCC6538, Bacillus subtilis var spizizinii ATCC6633, Enterococcus faecalis ATCC29212 and Pseudomonas aeruginosa ATCC 9027 were screened by the agar disk diffusion method. Ethyl alcohol (70\%) was used as negative control.

\section{RESULTS AND DISCUSSION}

After the incubation period and the occurrence of the inhibition zones the results revealed that the $70 \%$ ethylic alcohol mushroom extracts have significant inhibitory activities in particular against Bacillus subtilis subsp. spizizinii, Escherichia coli and Staphylococcus aureus bacteria. Between all samples tested P. eryngii Pe1 mushroom extract had strongly inhibitory effect on Bacillus subtilis 


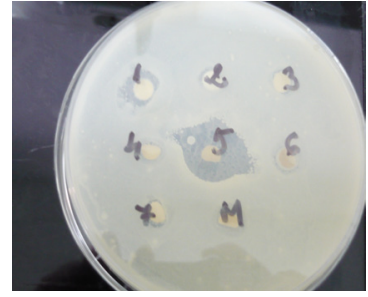

Figure 1. Antimicrobial activities of extracts against Bacillus subtilis subsp. spizizinii

1. L. edodes Lem 52; 2. L. edodes Lem 51; 3. L. edodes Leb; 4. Lentinus edodes Les; 5. P. eryngii Pe1; 6. P. eryngii Peg; 7. P. eryngii $\mathrm{Pe} 2, \mathrm{M}=$ control $(70 \%$ alcohol $)$.

subsp. spizizinii and promoted the formation of the largest inhibition zone (> $20 \mathrm{~mm}$ ) (Fig. 1).

The data relating to the antimicrobial activities of the mushroom extracts is summarized in Table 1.

Reports of other researchers indicating that the antimicrobial activity may arise from the genetic structure of mushroom species, physical, biochemical constituents, chemical differences of mushroom extracts, solvents and test microorganisms (Akyuz et al., 2010; Braga et al., 2011).

\section{CONCLUSION}

The results of present investigation revealed that the ethanol extracts of some edible mushrooms isolates showed biopharmaceutical potentiality as antimicrobial agents against pathogenic microorganisms. Also, the mushroom extracts can be an effective natural product for food and pharmaceutical industrial applications.

\section{Acknowledgements}

This work was made with the support of the Ministry of Agriculture and Rural Development - Romania, through the Rural Development Programme- ADER 2014-2020.

\section{REFERENCES}

1. Akyuz M, Onganer AN, Erecevit P, Kirbag S (2010). Antimicrobial Activity of some Edible Mushrooms in the Eastern and Southeast Anatolia Region of Turkey. GU J Sci, 23(2):125-130.

2. Braga K, Kasuya MC, Vanetti MCD (2011). Antimicrobial activity and mineral composition of shiitake mushrooms cultivated on agricultural waste. Braz. Arch. Biol. Technol. (54)5 991-1002.

3. Zhiming F, Liu Y, Zhang Q (2016). A Potent Pharmacological Mushroom: Pleurotus eryngii. Fungal Genom Biol. 6(1): 1-5. 